\title{
The Coupling of Boundary Element and Finite Element Methods 'for a Nonlinear Exterior Boundary Value Problem ${ }^{1}$ )
}

\author{
G. N. Gatica and G. C. Hsiao
}

Dedicated to Prof. Dr. Dr.h. c. Solomon G. Mikhlin on the occasion of his 80th birthday

In der Arbeit werden 'die Methoden der beschränkten und der finiten Elemente gekoppelt und auf das äußere Dirichletsche Problem für eine Klasse nichtlinearer elliptischer Gleichungen zweiter Ordnung in Divergenzform angewandt. Besondere Betonung erfährt die Formulierung in Variationsform und die mathematische Begründung der Kopplungsprozedur. Insbesondere werden hinreichende Bedingungen an die Koeffizienten der Gleichung angegeben, unter denen mit Hilfe der Methode der monotonen Operatoren die Existenz einer Lösung und deren Unität folgen.

- Работа посвящена применению соединсния методов ограниченных й финитных элемен:'тов к внешней задаче Дирихле для одного класса нелинеїных эллинтических уравнений второго порядка в дивергентной форме. Особ̄ое значение придастся вариационной формулировке и математическому обоснованию соединительнои пропедуры. В частности, даются достаточные условия на козффициенты уравнения из которых методом монотонных операторов следуют существование и единственность решения.

This paper is concerned with an application of the coupling of the boundary element and the finite element methods to an exterior. Dirichlet problem for a class of nonlinear second-order elliptic equations in divergence form. Emphasis will be placed upon the variational formulation and the mathematical foundations of the coupling procedure. In particular, sufficient conditions are given for the coefficients of the equation from which existence and uniqueness results are established by the theory of monotone operators:

\section{Introduction}

Both the boundary element and the finite element methods are now recognized as general approximation processes which are applicable to a wide variety of engineering problems. The boundary element method is, better suited to problems in which the domain extends to infinity but.is usually confined to regions in which the governing equations are linear and homogeneous. On the other hand, the finite element method is restricted to problems in bounded domains but is applicable to problems in which the material properties are not necessarily homogeneous and nonlinearity may occur. Therefore, these two methods are complementary to each other, and thus, in recent years there have been increasing efforts to develop variational procedures especially for exterior problems by taking the advantages of the individual method. This leads us to the coupling of the boundary element and the finite element methods (see Hsiao [6]). Noticeable success of this approach may be found in CostabeL [2], HAN [5], Hsiao and Porter [8], Johinson and Nedelec [10], MacCaMy and Marin [11],

1) This research was supported in part by the Center for Advinced Study of the University of Delaware. 
and WENDLaND [16, 17], to name a few. However; all these works deal with only linear problems. The purpose of this paper is to discuss the feasibility of applying this approach to genuine nonlinear problems.

1. Specifically, we let' $\Omega_{0}$, be a bounded, simply connected domain in $\mathbf{R}^{3}$ with smooth boundary $\Gamma_{0}$. Let $\Omega^{-}$be the annular region bounded by $\dot{\Gamma}_{0}$ and another smooth closed surface $\Gamma$. We denote by $\Omega^{+}$the complement of $\Omega_{0} \cup \bar{\Omega}^{-}$. For any function $v$ on $\Omega^{-} \cup \Omega^{+}$, we write $v^{ \pm}$for its limits on $\Gamma$ from $\Omega^{ \pm}$. Then given smooth functions $f$ on $\Omega^{-}$and $g$ on $\Gamma_{0}$, we consider the boundary value problem: Find $u$ such that

$$
\begin{aligned}
& -\sum_{i=1}^{3} \frac{\partial}{\partial x_{i}} a_{i}(x, \nabla u(x))=f(x) \text { in } \Omega^{-} ; \quad \Delta u=0 \text { in } \Omega^{+}, \\
& u=g \text { on } \Gamma_{0}, \\
& u^{-r}=u^{+}, \quad \sum_{i=1}^{3} a_{i}\left(x, \nabla u^{-}(x)\right) n_{i}=\left(\frac{\partial u}{\partial n}\right)^{+}(x) \text { on } \Gamma \\
& u(x)=O\left(|x|^{-1 j} \quad \text { as }|x| \rightarrow+\infty\right.
\end{aligned}
$$

where $\mathrm{n}=\left(n_{1}, n_{2}, n_{3}\right)$ is the outer normal to $\Gamma$. The nonlinear coefficients $a_{i}$ will satisfy certain regularity conditions: to be specified later. Explicit examples of non- linearities of this type generally appear in some subsonic flow problems (see, e.g. Feistauer [3]). Our goal here is to solve the problem (1.1) by the coupling procedure as in HAN [5] and CostaBEL' [2] for linear problems.

The plan of this paper is a follows. In-Section 2 , we will convert (1.1) to a nonlocal boundary problem in $\Omega^{-}$. This contains a nonlocal boundary condition which relates the unknown function and its normal derivative on the boundary $\Gamma$ from 'Green's formula. In Section 3, we give a weak formulation for the nonlocal boundary problem and reduce it to an equivalent operator equátion form. Existence and uniqueness of the solution of this operator equation will be established in Section 4 by the theory of monotone operators. Finally in Section 5, we present a Galerkin procedure for the operator equation and provide an error estimate of Cea's type.

\section{The nonlocal boundary problem}

To reduce (1.1)-to a nonlocal boundary problem, we need some results from potential theory. Let $\gamma(x, y)=(4 \pi|x-y|)^{-1}$ be the fundamental solution for the threedimensional.Laplacian. Then from Green's theorem we have the representation

$$
u(x)=\int_{i} u^{+}(y) \frac{\partial}{\partial n_{y}} \gamma(x, y) d s_{\nu}-\int_{\Gamma}\left(\frac{\partial u}{\partial n}\right)^{+}(y) \gamma(x, y) d s_{y}, \quad x \in \Omega^{+}
$$

from which we arrive at the integral equations on $\Gamma$ :

$$
\begin{aligned}
& u^{+}=\left(\frac{1}{2} \mathbf{I}+\mathbf{K}\right) u^{+}-\mathbf{V}(\partial u / \partial n)^{+} \\
& (\partial u / \partial n)^{+}=-\mathbf{W} u^{+}+\left(\frac{1}{2} \mathbf{I}-\mathbf{K}^{\prime}\right)(\partial u / \partial n)^{+}
\end{aligned}
$$

Here $K, V, \dot{W}$ and $K^{\prime}$ are the boundary integral operators of the douible, simple, hyper- singular and adjoint of the double layer potentials, respectively: For $x \in \Gamma$, they are 
defined by

$$
\begin{gathered}
\mathbf{K} \sigma(x)=\int_{\Gamma} \sigma(y) \frac{\partial}{\partial n_{y}} \gamma(x, y) d s_{\nu}, \quad \mathbf{V} \sigma(x)=\int_{\Gamma} \gamma(x, y) \sigma(y) d s_{\nu}, \\
\mathbf{W} \sigma(x)=-\frac{\partial}{\partial n_{x}} \int_{\Gamma} \sigma(y) \frac{\partial}{\partial n_{y}} \gamma(x, y) d s_{\nu}, \cdots \\
\vdots \\
\mathbf{K}^{\prime} \sigma(x)=\int_{\Gamma} \frac{\partial}{\partial n_{x}} \gamma(x, y) \sigma(y) d s_{\nu},
\end{gathered}
$$

These are four basic boundary integral operatoris (see MICHurn [13]) whose properties will be discussed later. Now we use interface conditions from (1.1). We have $u^{+}=u^{-}$, and if we set $\sum_{i=1}^{3} a_{i}\left(x, \nabla \dot{u}^{-}(x)\right) n_{i}=\sigma(x)$, then $(\partial u / \partial n)^{+}=\sigma$. We substitute into (2.2) to obtain, on $\Gamma$,

$$
\begin{aligned}
& \left(\frac{1}{2} \mathbf{I}-\mathbf{K}\right) u^{-}+\mathbf{V} \sigma=\ddot{0}, \\
& \sum_{i=1}^{3} \dot{a}_{i}\left(\cdot ; \nabla u_{-}^{-(\cdot))} n_{i}=-\mathbf{W} u^{-}+\left(\frac{1}{2} \mathbf{I}-\mathbf{K}^{\prime}\right) \sigma .\right.
\end{aligned}
$$

These formulae lead us to, the nonlocal boundary problem: Find $(u, \sigma)$ such that

$$
\begin{aligned}
& \quad-\sum_{i=1}^{3} \cdot \frac{\partial}{\partial x_{i}} a_{i}(x, \nabla u(x))=f(x) \quad \text { in } \Omega^{-} ; \quad u r_{r_{0}}=g, \\
& \therefore \sum_{i=1}^{3} a_{i}\left(\cdot, \nabla u^{-}(\cdot)\right) n_{i}=-\mathbf{W} u^{-}+\left(\frac{1}{2} \mathbf{I}-\mathbf{K}^{\prime}\right) \sigma \quad \text { on } \Gamma, \\
& \therefore\left(\frac{1}{2} \mathbf{I}-\mathbf{K}\right) \dot{u}^{-}+\mathbf{V} \sigma=0 \quad \text { on } \Gamma .
\end{aligned}
$$

It is easy to see from the above derivation that the nonlocal boundary problem (2.5) is equivalent to the problem (1.1) in the sense that if $(u, \sigma)$ satisfies (2.5) and if we set, from Green's formula (2.1),

$$
u(x)=\int_{\Gamma} u^{-}(y) \frac{\partial}{\partial n_{y}} \gamma(x, y) d s_{\nu}-\int_{\Gamma} \sigma(y) \gamma(x, y) d s_{y}, \quad x \in \Omega^{+}
$$

then $u$ satisfies (1.1) with $\sum_{i=1}^{3} a_{i}\left(x, \nabla u^{-}(x)\right) n_{i}=\sigma(x)$ on $\Gamma$. Of course, the statement here can be made more precise if one introduces appropriate solution spaces (see Gatica [4]). We remark that the boundary conditions on $\Gamma$ are nonlocal conditions, since the values $u^{-}$over the entire boundary $\Gamma$ are needed in order to compute $\therefore \sum_{i=1}^{3} a_{i}\left(x, \nabla u^{-}(x)\right) n_{i}$ at a single point $x \in \Gamma$. Clearly, one may also consider other types of nonlocal conditions, and in principle, the nonlocal boundary problem (2.5) can be treated numerically by any conventional scheme, since it is a problem over the finite region. We will adopt the Galerkin procedure and lead to the coupling of the boindary element and finite element methods. 


\section{The weak formulation}

In what follows, for $m$ integer, $s$ real, let $\hat{H}^{m}\left(\Omega^{-}\right)$and $H^{s}(\Gamma)$ denote the usual Sobolev spaces equipped with norms $\|\cdot\|_{H^{m}\left(\Omega^{-}\right)}$and $\|\cdot\|_{H^{\prime}\left(\Gamma_{)}\right)}$, respectively. Furthermore, for $m>0$, let $|\cdot|_{H^{m i}\left(\Omega_{-)}\right)}$denote the seminorm,

$$
|u|_{\left.H^{m(S 2)}\right)}=\left\{\sum_{|\alpha|=m} \int_{\Omega^{-}}\left|D^{a} u\right|^{2} d x\right\}^{1 / 2}
$$

and let $\langle\cdot, \cdot\rangle$ denote the duality pairing between $H^{s}(\Gamma)$ and $H^{-8}(\Gamma)$ with respect to the $L^{2}(I)$-innér product,

$$
\langle\sigma, \chi\rangle=\int_{\Gamma} \sigma(t) \chi(t) d t \quad \forall(\sigma, \chi) \in H^{s}(\Gamma) \times H^{-s}(\Gamma) .
$$

W.e also introduce the subspace $H_{\Gamma_{0}}^{1}\left(\Omega^{-}\right)$of $H^{1}\left(\Omega^{-}\right)$defined by

$$
H_{\Gamma_{0}}^{1}\left(\Omega^{-}\right)=\left\{v \in H^{1}\left(\Omega^{-}\right):\left.v\right|_{\Gamma_{0}}=\dot{0}\right\} .
$$

It is easy to verify that $|v|_{H^{1}\left(\Omega^{-}\right)}$is equivalent to the norm $\|v\|_{H^{1}\left(\Omega^{-}\right)}$for all $v \in H_{\Gamma_{0}}^{1}\left(\Omega^{-}\right)$, i.e., there exists a constant $C>0$ such that.

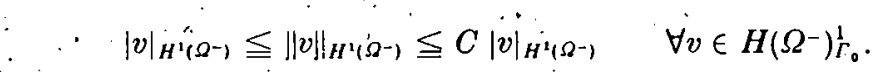

For smooth $\Gamma$, the following results are well known (see, e.g., Hsiao [7], and Hsisio and WENDLAND [9]).

Lem ma 1: For ' $C^{\infty}$ boundary $\Gamma$, the boundary integral operators defined by (2.3)

$$
\begin{array}{ll}
\mathbf{V}: H^{s+1 / 2}(\Gamma) \rightarrow H^{s+1 / 2}(\Gamma), & \mathbf{K}: H^{s+1 / 2}(\Gamma) \rightarrow H^{s+3 / 2}(\Gamma), \\
\mathbf{K}^{\prime}: H^{s-1 / 2}(\Gamma) \rightarrow H^{s+1 / 2}(\Gamma), & \mathbf{W}: H^{s+1 / 2}(\Gamma) \rightarrow H^{s-1 / 2}(\Gamma)
\end{array}
$$

are conitinuous for any $s \in$ R. Moreover, there are constants $\mu>0$ and $\nu \geqq 0$ such that

$\therefore \quad\left\langle\langle\mathbf{V} \sigma, \sigma\rangle \geqq \mu_{1}\|\sigma\|_{H^{-1 / 2}(\Gamma)}^{2} \quad \dot{\forall} \sigma \in H^{-1 / 2}(\Gamma)\right.$

and

$$
\langle\sigma, \mathrm{W} \sigma\rangle \geqq \mu\|\sigma\|_{H^{1 / 2}(\Gamma)}^{2}-v\|\sigma\|_{H^{\circ}(\Gamma)}^{2} \quad \forall \sigma \in \dot{H}^{1 / 2}(\dot{\Gamma}) .
$$

In fact, from (3.5) it is not difficult to show that

$$
\langle\sigma, \mathbf{W} \sigma\rangle \geqq 0 \quad \cdot \forall \sigma \in H^{1 / 2}(\Gamma) .
$$

These results will be needed later. -

Now for the weak formulation, we multiply the partial differential equation in (2.5) by any function $v \in H_{\Gamma_{0}}^{1}\left(\Omega^{-}\right)$and apply the divergence theorem to yield

$$
\sum_{i=1}^{3} \int_{\Omega^{-}} a_{i}(x, \nabla u(x)) \frac{\partial v}{\partial x_{i}} d x+\left(v^{-} ; \mathbf{W} u^{-}-\left(\frac{1}{2} \mathbf{I}-\mathbf{K}^{\prime}\right) \sigma\right\rangle=\int_{\Omega^{-}} f v d x
$$

Similarly, we multiply the boundary integral equation in (2.5) by any test function i. $\in H^{-1 / 2}(\Gamma)$ and integrate over $\Gamma$ to obtain

$$
\langle\dot{\mathbf{V}} \sigma, \lambda\rangle+\left\langle\left(\frac{\mathbf{1}}{2} \mathbf{I}-\mathbf{K}\right) u^{-}, \lambda\right\rangle=0
$$


Equations (3.8) and (3.9) then lead us to the weak formulation: Given $(f, g) \in H^{-1}\left(\Omega^{-}\right)$ $\times H^{1 / 2}(\Gamma)$, find $(u, \sigma) \in H^{1}\left(\Omega^{-}\right) \times H^{-1 / 2}(\Gamma)$ such that $u-\tilde{g} \in H_{\Gamma_{0}}^{\prime}\left(\Omega^{-}\right)$and $(u, \sigma)$ satisfies

$$
\sum_{i=1}^{3} \int_{\Omega} a_{i}(x, \nabla u(x)) \frac{\partial v}{\partial x_{i}} d x+\mathbf{B}((u, \sigma) ;(v, \lambda))=\int_{\Omega^{-}} j v d x
$$

for all $(v, \lambda) \in H_{\Gamma_{0}}^{1}\left(\Omega^{-}\right) \times H^{-1 / 2}(\Gamma)$, where $\tilde{g} \in H^{1}\left(\Omega^{-}\right)$is an extension of $g$ with $\left.\tilde{g}\right|_{\Gamma_{0}}=g$, and $\mathbf{B}(\cdot, \cdot)$ is the bilinear form defined by

$$
\begin{aligned}
\mathbf{B}((u, \sigma),(\dot{v}, \lambda))^{\prime}= & \left\langle v^{-}, \mathbf{W} u^{-}\right\rangle-\left\langle v^{-},\left(\frac{1}{2} \mathbf{I}-\mathbf{K}^{\prime}\right) \sigma\right\rangle \\
& +\langle\mathbf{V} \sigma, \lambda\rangle+\left\langle\left(\frac{1}{2} \mathbf{I}-\mathbf{K}\right) u^{-}, \lambda\right\rangle
\end{aligned}
$$

for all $(u-\tilde{g}, \sigma),(v, \dot{\lambda}) \in H_{\Gamma_{0}}^{\dot{1}}\left(\Omega^{-}\right) \times H^{-1 / 2}(\Gamma)$.

In order to reduce (3.10) to an equivalent operator equation form, we need now to impose some conditions on the nonlinear coefficients $a_{i}$. We assume that $a_{i}: Q^{-}$ $\times \mathbf{R}^{3} \rightarrow \mathbf{R}^{\prime}$ and satisfies the conditions:

(H.1) Carathéodory conditions: The, function $a_{i}(\cdot, \alpha)$ is measurable in $\Omega^{-}$for all $\alpha \in \mathbf{R}^{3}$ and $a_{i}(x, \cdot)$ is continuous in $\mathbf{R}^{3}$ for almost all $x \in \Omega^{\prime}$.

(H.2) Growth condition: There exists $\phi_{i} \in L^{2}\left(\Omega^{-}\right), i=1,2,3$, such that'

$$
\left|a_{i}(x, \alpha)\right| \leqq C\{1+|\alpha|\}+\left|\phi_{i}(x)\right|
$$

for all $\alpha \in \mathbf{R}^{3}$ and for almost all $x \in \Omega^{-}$. Here and in the sequel, $C$ is a generic constant.

We also define the Hilbert space $\mathbf{H}=H_{\Gamma_{0}}^{1}\left(\Omega^{-}\right) \times H^{-1 / 2}(\Gamma)$ with the product norm $\|(w, \sigma)\|_{\mathbf{H}}=\left\{\|w\|_{H^{1}\left(\Omega^{-}\right)}^{2}+\|\sigma\|_{\left.H^{-1 / 2}(\Gamma)\right\}^{1 / 2}}^{2}\right.$. Further, let $\mathbf{H}^{*}$ be the dual of $\mathbf{H}$, with the norm defined' by, $\|\cdot\|_{\mathbf{H}^{*}}=\sup \left\{[\cdot,(v, \lambda)] /\|(v, \lambda)\|_{\mathbf{H}}: 0 \neq(v, \lambda) \in \mathbf{H}\right\}$; where $[\because, \cdot]$ denotes the duality pairing on $\mathbf{H}^{*} \times \mathbf{H}$ : Then, by $(\mathbf{H} .1)$ and $(\mathbf{H} .2)$ we can introduce a nonlinear operator ' $\mathbf{T}: \mathbf{H} \rightarrow \mathbf{H}^{*}$ defined by

$$
[\mathbf{T}(w, \sigma),(v, \lambda)]=\sum_{i=1}^{3} \int_{\Omega^{-}} a_{i}(x, \nabla(w+\tilde{g})(x)) \cdot \frac{\partial v}{\partial x_{i}} d x+\mathbf{B}((w ; \sigma),(v, \lambda))
$$

for all $(w, \sigma),(v, \lambda) \in \mathbf{H}$. Consequently, if we set, in the weak formulation, $\dot{w}:=u-\vec{g}$ $\epsilon \dot{H}_{\Gamma_{0}}^{1}\left(\Omega^{-}\right)$, alternatively, we may write (3.10) in the form of an operator equation for the unknown $(w, \sigma)$ :-

$$
\mathbf{T}(w, \sigma)=\mathbf{F},
$$

where, $\mathbf{F} \in \mathbf{H}^{*}$. is defined by

$$
\dot{\mathbf{F}}(v, \lambda)=\int_{\Omega^{-}} f v d x-\mathbf{B}((\bar{g}, 0),(v, \lambda))
$$

We remark that the bilinear form $\mathbf{B}$ is bounded as a consequence of Lemma 1 and the trace theorem. The operator equation form (3.13) then allows us to dicuss the solvability of the rariational equation $(3.10)$ by the theory of monotone-operators. 


\section{Existence and uniqueness}

To apply the theory of monotone operators, following NEČs [14] and ODEN [15], we need to make further assumptions on the nonlinear coefficients $a_{i}$ in (3.10). We consider the following conditions:

(H.3) Coerciveness condition: There exist a constant $C_{1}>0$ and a function $C_{2}$ $\in L^{1}\left(\Omega^{-}\right)$such that $\sum_{i=1}^{3} a_{i}(x, \alpha) \alpha_{i} \geqq C_{1}|\alpha|^{2}-C_{2}(x)$ for all $\alpha=\left(\alpha_{1}, \alpha_{2}, \alpha_{3}\right) \in \mathbf{R}^{3}$ and for almost all $x \in \Omega^{-}$.

(H.4) Monolony condition:

$$
\sum_{i=1}^{3}\left(a_{i}(x, \boldsymbol{\alpha})-a_{i}\left(x, \alpha^{\prime}\right)\right)\left(\alpha_{i}-\alpha_{i}^{\prime}\right) \geqq 0
$$

for all $\boldsymbol{\alpha}, \dot{\alpha}^{\prime}, \in \mathbf{R}^{3}$ and for almost all $x \in \Omega^{-}$.

(H.5) Strict monotony, condition:

$$
\sum_{i=1}^{3}\left(a_{i}(x, \alpha)-a_{i}\left(x, \alpha^{\prime}\right)\right) /\left(\alpha_{i}-\alpha_{i}^{\prime}\right)>0{ }^{\prime}
$$

for all $\alpha \neq \alpha^{\prime} \in \mathbf{R}^{3}$ and for almost all $x \in \Omega^{-}$.

- (H.6) Strongly monotone condition: There exists a constant $\mu \geq 0$ such that

$$
\sum_{i, j=1}^{3}\left(\partial a_{i}(x, \alpha) / \partial \alpha_{j}\right) \xi_{i} \xi_{j} \geqq \mu \sum_{i=1}^{3} \xi_{i}^{2}
$$

for all $\xi=\left(\xi_{1}, \xi_{2}, \xi_{3}\right), \dot{\alpha}=\left(\alpha_{1}, \alpha_{2}, \alpha_{3}\right) \in \mathbf{R}^{3}$ and for almost all $x \in \Omega^{-}$.

Clearly these conditions are not mutually exclusive. Hence our main results can be summarized in the following theorems depending on the assumptions on $a_{i}$.

Theorem 1: Suppose that the coefficients $a_{i}$ salisfy the assumptions (H.1)-(H.4). Let $\mathbf{T}: \mathbf{H} \rightarrow \mathbf{H}^{*}$ be the operator defined by (3.12) and let $\mathbf{F} \in \mathbf{H}^{*}$ be the bounded linear functional defined by (3.14). Then there exists a solution $(w, \sigma) \in \mathbf{H}$ of the equation (3.13),, and the solution is unique if (H.5) is sutisfied.

Theorem 2: Under the assumption (H.1) and (H.2) the operator $\mathbf{T}$ is continuous and bounded. Furthermore, if (H.6) holds, then $\mathrm{T}$ is strongly monotone; i.e., there exists a constant $\mu>0$ such that

$$
[\mathbf{T}(w ; \sigma)-\mathbf{T}(v, \lambda),(w, \sigma)-(v, \dot{\lambda})] \geqq \mu\|(w, \sigma)-(v, \dot{\lambda})\|_{\mathbf{H}}^{2}
$$

for all $(w, \sigma),(v, \lambda) \in \mathbf{H}$, and hence $\mathbf{T}$ is coercive on $\mathbf{H}$; i.e., .

$$
\lim _{\|(w, \sigma)\|_{\mathbf{H} \rightarrow+\infty}[\mathbf{T}(w, \sigma),(w, \sigma)] /\|(w, \boldsymbol{\sigma})\|_{\mathbf{H}}=+\infty}
$$

- We remark that Theorem 2 implies that the equation (3.13) has a unique solution.

The proofs, of these theorems are tedious and lengthy, but the arguments here are straightforward if one is familiar with the theory of monotone operators (see, e.g., NEČAS [14]). To facilitate the proofs, let us first make some observations: We note that the boundary operators corresponding to $B$ in the definition of the nonlinear operator $T$ of (3.12) possess all the nice properties. In particular, we see that in addition to being bounded,

$$
|\mathbf{B}((w, \ddot{\sigma}),(v, \lambda))| \leqq C\|(w, \boldsymbol{\sigma})\|_{\mathbf{H}}\|(v, \lambda)\|_{\mathbf{H}}
$$


for all $(w, \bar{\sigma}), \dot{(}(v, \lambda) \in \mathbf{H}, \mathbf{B}$ satisfies the inequality,

$$
\dot{\mathbf{B}}((w, \sigma),(w, \sigma)) \geqq \mu\|\sigma\|_{H^{-1 / 2}(\Gamma)}^{2} \quad \forall(w, \sigma) \in \mathbf{H},
$$

which follows from (3.11), (3.5) and (3.7). Hence the operator $\mathbf{T}$ is completely dominated by the nonlinear term in $\mathbf{T}$, i.e., the Nemytsky operator. $\mathbf{A}_{i}$, defined by

$$
\left(\mathbf{A}_{i} w\right)(x)=a_{i}(x, \nabla(w+\tilde{g})(x)) .
$$

The assumptions (H.1) and (H.2) simply imply that $\mathbf{A}_{i}$ is a continuous map from $H^{1}\left(\Omega^{-}\right)$into $L^{2}\left(\Omega^{-}\right)$and the inequality

$$
\left\|\mathbf{A}_{\mathbf{i}} w\right\|_{L^{2}\left(\Omega^{-}\right)}^{2} \leqq C\left\{\operatorname{vol}\left(\Omega^{-}\right)+|w+\tilde{g}|_{H^{1}\left(\Omega^{-}\right)}^{2}+\left\|\phi_{i}\right\|_{L^{\prime}\left(\Omega^{-}\right)}^{2}\right\}
$$

holds for all $w \in H_{r_{0}}^{1}\left(\Omega^{-}\right)$. As a consequence of $(4.3),(4.6)$, we have the following lemma.

Lemma 2: Under the assumptions"(H.1) and (H.2), the operator $\mathbf{T}: \mathbf{H} \rightarrow \mathbf{H}^{*}$ has the following properties:

(i) $\mathbf{T}$ is bounded.

(ii) There exists a constant $M_{0}>0$ such that $\left\|\mathbf{A}_{\mathbf{i}} w\right\|_{L^{2}\left(\Omega^{-}\right)} \leqq C\|(w, \sigma)\|_{\mathbf{H}}$ for all $(w, \sigma) \in \mathbf{H},\|(w, \sigma)\|_{\mathbf{H}} \geqq M_{0}$.

(iii) $\mathbf{T}$ is continuous.

It is easy to verify that the constant $M_{0}$ in (ii) is given by .

$$
M_{0}=\left\{\operatorname{vol}\left(\Omega^{-}\right)+|\tilde{g}|_{H^{1}\left(\Omega^{-}\right)}^{2}+\sum_{i=1}^{3}\left\|\phi_{i}\right\|_{L^{2}\left(\Omega^{-}\right)}^{2}\right\}^{1 / 2} .
$$

The assumption (H.3) naturally gives the coerciveness property of $\mathbf{T}$. (4.2) as can be seen in the following. We may write from (3.13),

$$
\begin{aligned}
& \sum_{i=1}^{3} \int_{\Omega^{-}} a_{i}(x, \nabla(w+\tilde{g})(x)) \cdot \frac{\partial w}{\partial x_{i}} d x \\
& =\sum_{i=1}^{3} \int_{\Omega^{-}} a_{i}(x, \nabla(w+\tilde{g})(x)) \frac{\partial}{\partial x_{i}}(w+\tilde{g}) d x \\
& \quad-[\mathbf{T}(w, \sigma),(\tilde{g}, 0)]+\mathbf{B}((w, \sigma),(\tilde{g}, 0)),
\end{aligned}
$$

and hence from (H.3) together with (4.3) and (ii) in Lemma 2, we have

$$
\begin{aligned}
& \sum_{i=1}^{3} \cdot \int_{\Omega^{-}} a_{i}(x, \nabla(w+\tilde{g})(x)) \frac{\partial w}{\partial x_{\mathbf{i}}} d x \quad ; \\
& \geqq C_{1}|w+\bar{g}|_{H^{1}(\Omega-)}^{2}-\left\|C_{2}\right\|_{L^{1}(\Omega-)}-C\|(w, \sigma)\|_{\mathbf{H}}\|\bar{g}\|_{H^{\prime}(\Omega-)}
\end{aligned}
$$

for all $(w, \sigma) \in \mathbf{H}$ with $\|(w, \sigma)\|_{\mathbf{H}} \geqq M_{0}$. Consequently, we'deduce from (3.12), (3.4) and (4.4),

$$
\frac{[\mathbf{T}(w, \sigma),(w, \sigma)]}{\|(w, \sigma)\|_{\mathbf{H}}} \geqq \tilde{C}_{1}\|(w, \sigma)\|_{\mathbf{H}}-C\|\tilde{g}\|_{H^{\prime}\left(\Omega^{-}\right)}-\left\|C_{2}\right\|_{L^{1}\left(\Omega^{-}\right)}\|(w, \sigma)\| \overline{\mathbf{H}}^{\mathbf{l}}
$$

for all $(w, \sigma) \in \mathbf{H}$ with $\|(w, \sigma)\|_{\mathbf{H}} \geqq M_{\mathbf{0}}$. This proves that $\mathbf{T}$ is coercive on $\mathbf{H}$. 
Similarly, it is not difficult to verify that

$$
\begin{aligned}
& {[\mathbf{T}(w, \dot{\sigma})-\mathbf{T}(v, \dot{\lambda}),(w, \sigma)-(v, \bar{\lambda})]} \\
& =\mathbf{B}((w, \sigma)-(v, \hat{\lambda}),(w, \sigma)-(v, \lambda)) \\
& \quad+\sum_{i=1}^{3} \int_{\partial^{-}}\left\{a_{i}(x, \nabla \tilde{w}(x))-a_{i}(x, \nabla \tilde{v}(x))\right\}\left\{\frac{\partial \dot{w}}{\partial x_{i}}-\frac{\partial \tilde{v}}{\partial x_{i}}\right\} \ddot{d} \dot{x}
\end{aligned}
$$

with $\bar{w}=w+\bar{g}$ and $\bar{v}=v+\tilde{g}$. Because of (4.4); the monotonicity conditions will :be determined by the second term on the right-hand side of the above equality and thus by the conditions (H.4) and (H.5).'

- To complete the proof of 'Theorem' 1 , we remark that the continuity of 'T implies the hemicontinuity of $\mathbf{T}$, i.e., the mapping

$$
\mathbf{R} \ni t \rightarrow[\mathbf{T}((w, \sigma)+t(p, \delta)),(v, \lambda)] \in \mathbf{R} .
$$

is continuous for all $(w, \sigma),(p, \delta), .(v, \lambda)^{\circ} \in \mathbf{H}$. Moreover, $\mathbf{T}$ has the property (see $N$ Ečs [14: Theorem 3.3.14]):

(M) Let $\left\{\left(\dot{w}_{n}, \sigma_{n}\right)\right\}$ be a sequence of $\mathbf{H}$. Suppose that $\left(w_{n}, \sigma_{n}\right)$ converges weakly to $(w, \sigma)$, and $\mathbf{T}\left(w_{n}, \sigma_{n}\right)$ converges weakly to $\mathbf{F}$ with $\lim \sup \left[\mathbf{T}\left(w_{n}, \sigma_{n}\right),\left(w_{n}, \sigma_{n}\right)\right] \leqq[\mathbf{F},(w, \sigma)]$, then $\mathbf{T}(w ; \sigma)=F$.

$n \rightarrow+\infty$

We also note that the continuity of $\mathbf{T}$ implies, clcarly, the demicontinuity of $\mathbf{T}$, namely, if $\left(w_{n}, \sigma_{n}\right)$ converges to $(w ; \sigma)$, then $\mathbf{T}\left(w_{n}, \sigma_{n}\right)$ converges weakly to $\mathbf{T}(w, \dot{\sigma})$. Now, property (M), coerciveness, demicontinuity and boundedness of $\mathbf{T}$ implies that. $\mathbf{T}$ is surjective (see Nectas [14, Theorem 3.3.6]). The rest of the proof follows easily

To complete the proof of Theorem 2, it remains to show that under $\left(H_{j} 6\right), T$ satisfies the stiongly monotone condition (4.1), since the coerciveness of $\mathrm{T}$ is a simple consequence of (4.1). We proceed as follows: We note that we may write

$$
a_{i}(x, \nabla \tilde{w}(x))-a_{i}(x, \nabla \tilde{v}(x))=\int_{0}^{1} \sum_{j=1}^{3} \frac{\partial}{\partial \alpha_{j}} a_{i}(x, \alpha(x ; t))\left\{\frac{\partial \tilde{w}}{\partial x_{j}}-\frac{\partial \tilde{v}}{\partial x_{j}}\right\} d t
$$

with $\alpha(\dot{x}, t)=\nabla \tilde{v}(x)+t(\dot{\nabla} \tilde{w}(x)-\nabla \tilde{v}(x))$. Then $(\dot{H} .6)$ yields

$$
\begin{aligned}
& \sum_{i=1}^{3} \int_{\Omega=}\left\{a_{i}(x, \nabla \tilde{w}(x))-a_{i}(x, \nabla \dot{v}(x))\right\}\left\{\frac{\partial \tilde{w}}{\partial x_{i}}-\frac{\partial \bar{v}}{\dot{x}_{i}}\right\} d x \\
& \geq \mu \sum_{i=1}^{3} \int_{\Omega}\left\{\frac{\partial \bar{w}}{\partial x_{i}}-\frac{\partial \bar{v}}{\partial x_{i}}\right\}^{2} d x
\end{aligned}
$$

The result then follows from (3.4), (4.4) and (4.7)

\section{Galerkin approximations}

To formulate the Galerkin approximations of the solution of (3.15), we let $\left\{\mathbf{H}_{h}\right\}_{h \in S}$ be a family of finite-dimensional subspaces of $\mathbf{H}$ such that $\cup\left\{\mathbf{H}_{h}: h \in S\right\}$ is dense in $\mathbf{H}$, where the parameter $h$ is in an index set $S$ and represents, without loss of generality, a measure of the size of the corresponding finite elements. Then the Galerkin approxi- 
mation of the solution $(\bar{w}, \sigma)$ of $(3.15)$ is defined as an element $\left(w^{h}, \sigma^{h}\right) \in \mathbf{H}_{h}$ satisfying the Galerkin equations

$$
\left[\mathbf{T}\left(w^{h}, \sigma^{\dot{h}}\right),\left(v^{h} ; \lambda^{h}\right)\right]=\left[\mathbf{F},\left(v^{h}, \lambda^{h}\right)\right]
$$

for all $\left(v^{n}, \lambda^{n}\right) \in \mathbf{H}_{h}$. Clearly, the existence, uniqueness as well as the convergence of the Galerkin approximations $\left(w^{n}, \sigma^{n}\right)$ of $(5.1)$ depend heavily on the assumptions of the nonlinear coefficients $a_{i}$ in. T. From Theorem 1 in Section 4, we know at least that there exists a unique solution $(w, \sigma) \in \mathbf{H}$ of (3.13), provided (H.5) is fulfilled in addition to the assumptions (H.1)-(H.3): In this case, one cain show that there exists a unique Galerkin approximation $\left(w^{h}, \sigma^{h}\right)$ which has a subsequence converging only weakly to the exact solution $(w, \sigma)$ of $(3.13)$. On the other hand, the weak convergence of the Galerkin approximations can be improved by the strongly monotone condition (4.1). However, in order to obtain some kind of rate of convergence, an additional condition will be needed in contrast to the linear problems (see MICHIIN [12]). For this purpose, one will introduce a Lipschitz condition below. We recall. that $\mathbf{T}: \mathbf{H} \rightarrow \mathbf{H}^{*}$ is Lipschitz continuous if there exists a constant $k>0$ such that

$$
\therefore \mathbf{T}(w, \sigma)-\mathbf{T}(v, j)\left\|_{\mathbf{H}} \leqq k\right\|(w, \sigma)-(v, \lambda) \|_{\mathbf{H}} \text {. }
$$

for all $(w, \sigma),(v, \lambda) \in \mathbf{H}$. As will be.seen, a sufficient condition on the nonlinear coefficients $u_{i}$ to $\backslash$ ensure (5.2) is the following one:

(H.7) Lipschitz condition: The partial derivatives $\partial a_{i}(x, \alpha) / \partial \alpha_{j}{ }^{\prime}(i, j=1,2,3)$. satisfy the Carathéodory conditions (H.1) and there exists a.constant $C_{0}>0$ such that $\left|\partial a_{i}(x, \alpha) / \partial \alpha_{j}\right| \leqq C_{0 c}(i, j=1 ; 2,3)$ for all $\alpha \in \mathbf{R}^{3}$ and for almost all $x \in \Omega^{-}$.

To establish $(\dot{5} .2)$, it suffices to. show that the-Nemytsky operator $\boldsymbol{A}_{i}$ defined by (4.5) is Lipschitz continuous on $H^{1}\left(\Omega^{-}\right)$: We denote that under conditions $(\mathrm{H} .1)$ and (H.2), $\mathbf{A}_{\mathrm{i}}$ is a continuoús map from $H^{\mathrm{I}}\left(\Omega^{-}\right)$into $L^{2}\left(\Omega^{-}\right)$. For $\bar{w}=w+\bar{g}$ and $\bar{v}=$ $v+\bar{g}, \in H^{1}\left(\Omega^{-}\right)$, we have by the definition of $\mathbf{A}_{i}$,

$$
\begin{aligned}
\left\|\mathbf{A}_{i} w-\mathbf{A}_{i} v\right\|_{L^{\prime}\left(\Omega^{-}\right)}^{2} & =\int_{\Omega^{-}}\left|a_{i}(x, \grave{\nabla} \tilde{w}(x))-a_{i}(x, \nabla \tilde{v}(x))\right|^{2} d x \\
\quad & =\int_{\Omega^{-}}\left|\sum_{j=1}^{3} \int_{0}^{1} \frac{\partial}{\partial \alpha_{j}} a_{i}(x, \alpha(x, t))\left\{\frac{\partial \tilde{w}}{\partial x_{j}}-\frac{\partial \tilde{v}}{\partial x_{j}}\right\} d t\right|^{2} d x, .
\end{aligned}
$$

where $\alpha(x, \iota) \doteq \nabla \bar{v}(x)+\iota(\nabla \bar{w}(x)-\nabla \bar{v}(x))$. It follows from (H.7) that

$$
\left\|\mathbf{A}_{i} w-\cdot \mathbf{A}_{i} v\right\|_{L^{2}(\Omega-)}^{2} \leqq \ddot{3} C_{0}^{2}|\bar{w}-\bar{v}|_{\tilde{H}^{\prime}(\Omega-)}^{2} \leqq C\|w-v\|_{H^{1}(\Omega-)}^{2}
$$

which proves that $A_{i}: H^{1}\left(\Omega^{-}\right) \rightarrow L^{2}\left(\Omega^{-}\right)$is Lipschitz continuous. .

The following result concerning the error estimates of the Galerkin approximations can now be easily established.

Theorem 3: Suppose that the assumptions (H.1), (H.2), (H.6), and (H.7) are fulfilled. Let $(w, \sigma) \in \mathbf{H}$ and $\left(w^{h}, \sigma^{h}\right) \in \mathbf{H}_{h}$ be the unique solutions of the equations (3.13) and (5.1), respectively. Then, there exists a constant $C>0$ independent of h such thai

$$
\left\|(w, \sigma)-\left(w^{h}, \sigma^{h}\right)\right\|_{\mathbf{H}} \leqq C \inf _{\left(v^{n}, \lambda^{n}\right) \in \mathbf{H}_{h}}\left\|(w, \dot{\sigma})-\left(v^{n}, \lambda^{h}\right)\right\|_{\mathbf{H}} \cdot
$$

We remark that as in the case for linear problems, this simple, yet crucial estimate (5.3) shows that the problem of estimating the error between the solution $(\dot{w}, \sigma)$ and the Galerkin approximation $\left(w^{h}, \sigma^{h}\right)$ is reduced to a problem in the approximation 
theory. We further comment that for the numerical implementations, one generally does not solve (5.1) exactly. This leads us to consider the following modification of (5.1). To this end, let $\mathbf{T}_{h}: \mathbf{H}_{h} \rightarrow \mathbf{H}_{h}{ }^{*}$ be an operator that approximates $\mathbf{T}$ on the subspace $\mathbf{H}_{h}$, and let $\mathbf{F}_{h} \in \mathbf{H}_{h}{ }^{*}$ be an approximation of $\mathbf{F}$ on $\mathbf{H}_{h}$. Then we redefine a Galerkin approximation of the solution $(w, \sigma)$ of $(3.13)$ as an element $\left(w_{h}, \sigma_{h}\right) \in \mathbf{H}_{h}$ (if it exists) such that

$$
\cdots\left[\mathbf{T}_{h}\left(w_{h}, \sigma_{h}\right),\left(v^{h}, \lambda^{h}\right)\right]_{h}=\left[\mathbf{F}_{h}\left(v^{h}, \lambda^{h}\right)\right]_{h}
$$

for all $\left(v^{h}, \hat{\lambda}^{h}\right) \in \mathbf{H}_{h}$, where $[\cdot, \cdot]_{h}$ denotes the duality pairing on $\mathbf{H}_{h}{ }^{*} \times \mathbf{H}_{h}$. As far as the existence of a solution of (5.4) concerns, it suffices, from Theorem 1 , to assume that, $\mathbf{T}_{h}$ is a continuous, monotone and coercive operator on $\dot{\mathbf{H}}_{h}$. Since $\mathbf{H}_{h}$, is finitedimensional, the monotonicity of $\mathbf{T}_{h}$ implies that $\mathbf{T}_{h}$ is bounded (see ODEN [15: Theorem 27.3]). Similarly, from 'Theorem 2, we see that there exists a unique solution of (5.4) if $\mathbf{T}_{h}$ is continuous and strongly. monotone on $\mathbf{H}_{h}$. We now summarize these results in the following theorem.

Theorem' 4: Suppose that the coefficients $a_{i}$ satisfy the assumptions (H.1), (H.2)' (H.6) and (H.7). Let $\mathbf{F}_{h} \in \mathbf{H}_{h}{ }^{*}$ be an approximation of $\mathbf{F}$; and let $\mathbf{T}_{h}: \mathbf{H}_{h} \rightarrow \mathbf{H}_{h}{ }^{*}$ be an approximate operator of $\mathbf{T}$ with the properties:

(i) $\mathbf{T}_{h}$ is continuous.

(ii) $\mathbf{T}_{h}$ is uniformly strongly monotone, i.e., there exist constants $h_{0}>0$ and $\mu_{0}>0$, independent of h, such that

$$
\therefore \quad\left[\mathbf{T}_{h}\left(u_{h}, x_{h}\right)-\mathbf{T}_{h}\left(v^{h}, \lambda^{h}\right),\left(u_{h}, x_{h}\right)-\left(v^{h}, \lambda^{h}\right)\right]_{h} \geqq \mu_{\mathbf{0}}\left\|\left(\dot{u}_{h}, x_{h}\right) \div\left(v^{h}, \lambda^{h}\right)\right\|_{\mathbf{H}}^{2}
$$

for all $\left(u_{h}, x_{h}\right),\left(v^{h}, \hat{i}^{h}\right) \in \mathbf{H}_{h}$ and for äll $h \in\left(0, h_{0}\right)$.

T'hen (3:13) has a unique solution $(w, \sigma) \in \mathbf{H}$ and $(5.4)$ has a unique solution $\left(w_{h}, \sigma_{h}\right)$.

$\epsilon \mathbf{H}_{h}$. Moreover, the following estimates hold for all $h \in\left(0, h_{0}\right)$ :

$$
\begin{aligned}
& \left\|(w, \sigma)-\left(w_{h}, \sigma_{h}\right)\right\|_{\mathbf{H}} \leqq C\left\{\sup _{\left\{\begin{array}{c}
\left(z^{h}, \delta^{h}\right) \in \mathbf{H}_{h} \\
\left(z^{h}, \delta^{h}\right) \neq 0
\end{array}\right.} \frac{\left\|\left[\mathbf{F}_{h},\left(z^{h}, \delta^{h}\right)\right]_{h}-\left[\mathbf{F},\left(z^{h}, \delta^{h}\right)\right]\right\|}{\left\|\left(z^{h}, \delta^{h}\right)\right\|_{\mathbf{H}}}\right. \\
& +\inf _{\left(0^{n}, \lambda^{h}\right) \in \mathbf{H}_{h}}\left(\left\|(w, \sigma)-\left(v^{h}, \dot{\lambda}^{h}\right)\right\|_{\mathbf{H}}\right. \\
& \left.\left.+\sup _{\substack{\left(z^{h}, \delta^{h}\right) \in \mathbf{H}_{\boldsymbol{A}} \\
\left(z^{h}, \delta^{h}\right) \dot{+} \mathbf{0}}} \frac{\left[\left[\mathbf{T}\left(v^{h}, \lambda^{h}\right),\left(z^{h}, \delta^{h}\right)\right]-\left[\mathbf{T}_{h}\left(v^{h}, \lambda^{h}\right),\left(z^{h}, \delta^{h}\right)\right]_{h} \mid\right.}{\left\|\left(z^{h}, \delta^{h}\right)\right\|_{\mathbf{H}}}\right)\right\}
\end{aligned}
$$

- where $C>0$ is a,constant independent of $h$.

The proof of the inequality (5.5) follows in the same manner as for the linear problems (see, e.g., Ciarlet [1]). We omit the details.

To conclude this paper, we remark that in GatiCa [4], an explicit operator $\mathbf{T}_{h}$ will be given to possess the properties of (i) and (ii) in Theorem 4, and specific error estimates based on (5.5) will be also available for a family of finite element subspaces $\left\{\mathbf{H}_{h}\right\}_{h \in\left(0, h_{\bullet}\right)}$. 


\section{REFERENCES}

d. [1] Ciarlet, P. G.: The Finite Element' Method for Elliptic Problems. Amsterdam: North. Holland Publ. Comp. 1978.

[2] Costabel, M.: Symmetric methods for the coupling of finite elements and boundary elements. In: Boundary Elements 9, Vol. 1 (Ed.: C. A. Brebbia et al). Berlin-HeidelbergNew York: Springer-Verlag 1987, 411-420.

[3] Feistauer, M.: On the finite element approximation of a cascade flow problem. Numer. Math. 50 (1987), 655-684.

[4] Gatica, G.: On the coupling of boundary integral and finite element methods for nonlinear boundary value problems. Ph. D. Dissertation. Délaware: University of Delaware 1988.

[5] $\mathrm{H}_{\Lambda \mathrm{N}}, \mathrm{H}$.: A new class of variational formulations for the coupling of finite and boundary element methods. J. Comp. Math. (to appear).

[6] HsiaO, G. C.: The coupling of BEM and FEM - a brief review. In: Boundary Elements 10 (Ed.: C. A. Brebbia et al). Berlin-Heidelberg-New York: Springer-Verlag 1988. ,

[7] Hsiao, G. C.: On boundary integral equations of the first kind. J. Comp. Math. 7 (1989).

[8] Hsiao, G. C., and J. F. Porter: The coupling of BEM and FEM for the two-dimensional viścous flow problem. Appl. Anal. 27 (1988), 79-108.

[9] Hsiao, G. C., and. W. L. Wendand: The Aubin-Nitṣche lemma for integral equations. J. Int. Equ. 3 (1981), 299-315.

[10] Johnson, C., and J. C. NeDelec: On the coupling of boundary integral and finite element methods. Math. Comp. 35 (1980), $1063-1079$.

[11] MacCaisy, R. C., and S. P. Marin: A finite element method for exterior interface problems. Int. J. Math. and Math. Sci. 3 (1980), 311-350.

[12] Mickur, S. G.: Variationsmethoden der Mathematischen Physik. Berlin: AkademieVerlàg 1962 .

[13] Mic̀uls, S. G.: Partielle Differentialgleichungen in der Mathematischen Phyṣik. Berlin: Akademie-Verlag 1978.

[14] Nečss, J.: Introduction to the Theory of Nonlinear Elliptic Equations. New York: John Wiley \& Sons 1986.

[15] OdEn, J. T.: Qualitative Methods in Nonlinear Mechanics. Englewood Cliffs: PrenticeHall 1986.

[16] WendLAND, W. L.: On asymptotic error estimates for the combined BEM and FEM. In: - Innocative Methods in Engineering. Berlin--Heidelberg-New York: Springer-Verlag $1986,55-70$.

[17] WeNdand, W. L.: On asymptotic error estimates for the combined BEM and FEM. In: FEM and BEM from Engineering and Mathematical Point of View, to appear.

Manuskripteingang: 26. 09. 1988

\section{VERFASSER :}

- Dr. Gabriel N. Gatica

Departamento de Matematica

Universidád de Conception

Casilla 2017, Concepcion

Chile

Prof. Dr. George C. Hsiao

Department of Mathematical Sciences

University of Delaware

Newark, Delawure 197,16

U.S.A. 